\title{
Linear Port-Hamiltonian Descriptor Systems
}

\author{
Christopher Beattie* and Volker Mehrmann ${ }^{\dagger}$ and Hongguo $\mathrm{Xu}^{\ddagger}$ and Hans Zwart ${ }^{\S}$
}

October 5, 2018

\begin{abstract}
The modeling framework of port-Hamiltonian systems is systematically extended to linear constrained dynamical systems (descriptor systems, differential-algebraic equations) of arbitrary index and with time-varying constraints. A new algebraically and geometrically defined system structure is derived. It is shown that this structure is invariant under equivalence transformations, and that it is adequate also for the modeling of high-index descriptor systems. The regularization procedure for descriptor systems to make them suitable for simulation and control is modified to preserve the port-Hamiltonian form. The relevance of the new structure is demonstrated with several examples.
\end{abstract}

Keywords: port-Hamiltonian system, descriptor system, differential-algebraic equation, passivity, stability, system transformation, differentiation-index, strangeness-index, skew-adjoint operator.

AMS subject classification.: 93A30, 65L80, 93B17, 93B11.

\section{Introduction}

Modeling packages such as MODELICA (https://www.modelica.org/), MATLAB/SimulinK (http://www.mathworks.com) or SiMPACK [47] have come to provide excellent capabilities for the automated generation of models describing dynamical systems originating in different physical domains that may include mechanical, mechatronic, fluidic, thermic, hydraulic, pneumatic, elastic, plastic, or electric components [1, 17, 23, 44, 45]. Due to the explicit incorporation of constraints, the resulting systems comprise differential-algebraic equations (DAEs), also referred to as descriptor systems in the system theory context. Descriptor systems may contain hidden constraints, consistency requirements for initial conditions, and unexpected regularity requirements. Therefore, these models usually require further regularization to be suitable for numerical simulation and control, see [11, 30, 33]. Our focus will be on linear time-varying descriptor systems, as they typically arise from the linearization of

\footnotetext{
${ }^{*}$ Department of Mathematics, Virginia Tech, Blacksburg, VA 24061, USA. beattie@vt.edu. Supported by Einstein Foundation Berlin, through an Einstein Visiting Fellowship.

${ }^{2}$ Institut für Mathematik MA 4-5, TU Berlin, Str. des 17 . Juni 136, D-10623 Berlin, FRG. mehrmann@math.tu-berlin.de. Supported by Einstein Foundation Berlin via the Einstein Center ECMath and by Deutsche Forschungsgemeinschaft via Project A02 within CRC 1029 'TurbIn'

${ }^{3}$ Department of Mathematics, University of Kansas, Lawrence, KS 66045, USA. xu@math.ku.edu. Partially supported by Alexander von Humboldt Foundation and by Deutsche Forschungsgemeinschaft, through the DFG Research Center MATHEON Mathematics for Key Technologies in Berlin.

${ }^{4}$ University of Twente, Department of Applied Mathematics, P.O. Box 217, 7500 AE Enschede, The Netherlands. h.j.zwart@utwente.nl
} 
nonlinear DAE systems along a (non-stationary) reference trajectory, see [10]. These have the form

$$
\begin{aligned}
E(t) \dot{x}(t) & =A(t) x(t)+B(t) u(t), \\
y(t) & =C(t) x(t)+D(t) u(t),
\end{aligned}
$$

together with an initial condition $x\left(t_{0}\right)=x_{0}$. The coefficient matrices satisfy $E, A \in$ $C^{0}\left(\mathbb{I}, \mathbb{R}^{n, n}\right), B \in C^{0}\left(\mathbb{I}, \mathbb{R}^{n, m}\right), C \in C^{0}\left(\mathbb{I}, \mathbb{R}^{m, n}\right)$, and $D \in C^{0}\left(\mathbb{I}, \mathbb{R}^{m, m}\right)$, where we denote by $C^{j}(\mathbb{I}, \mathcal{X}) j \in\{0,1,2,3, \ldots\}$ the set of $j$-times continuously differentiable functions from a compact time interval $\mathbb{I}=\left[t_{0}, t_{f}\right] \subseteq \mathbb{R}$ to a vector space $\mathcal{X}$. If it is otherwise clear from the context, the argument $t$ of the coefficient functions is suppressed.

An important development in recent years has been to employ energy based modeling via bond graphs $[4,12]$. This has been implemented recently in 20-SIM (http://www . 20sim.com/), for example. The resulting systems have a port-Hamiltonian $(p H)$ structure, see e. g. [21, 27, $37,40,41]$, that encodes underlying physical principles such as conservation laws directly into the structure of the system model. The standard form for $p H$ systems appears as

$$
\begin{aligned}
& \dot{x}=(J-R) \nabla_{x} \mathcal{H}(x)+(B-P) u, \\
& y=(B+P)^{T} \nabla_{x} \mathcal{H}(x)+(S+N) u,
\end{aligned}
$$

where the function $\mathcal{H}(x)$ is the Hamiltonian typically describes the distribution of internal energy among energy storage elements of the system, $J=-J^{T} \in \mathbb{R}^{n, n}$ is the structure matrix describing energy flux among energy storage elements within the system; $R=R^{T} \in \mathbb{R}^{n, n}$ is the dissipation matrix describing energy dissipation/loss in the system; $B \pm P \in \mathbb{R}^{n, m}$ are port matrices, describing the manner in which energy enters and exits the system, and $S+N$, with $S=S^{T} \in \mathbb{R}^{m, m}$ and $N=-N^{T} \in \mathbb{R}^{m, m}$, describes the direct feed-through from input to output. It is necessary that

$$
W=\left[\begin{array}{ll}
R & P \\
P^{T} & S
\end{array}\right] \geq 0,
$$

where we write $W>0$ or $W \geq 0$ to assert that a real symmetric matrix $W$ is positive definite or positive semi-definite, respectively. Port-Hamiltonian systems generalize Hamiltonian systems, in the sense that conservation of energy for Hamiltonian systems is replaced by the dissipation inequality:

$$
\mathcal{H}\left(x\left(t_{1}\right)\right)-\mathcal{H}\left(x\left(t_{0}\right)\right) \leq \int_{t_{0}}^{t_{1}} y(t)^{T} u(t) d t .
$$

In the language of system theory, $\mathcal{H}(x)$ is a storage function associated with the supply rate, $y(t)^{T} u(t)$. The inequality, (4), describes a conservation property of the dynamical system that is termed passivity [8]. Note that $\mathcal{H}(x)$ defines a Lyapunov function for the unforced system, so minimal $\mathrm{pH}$ systems are implicitly Lyapunov stable [24]. Inequality (4) is an immediate consequence of (3) and holds even when the coefficient matrices $J, R, B, P, S$, and $N$ depend on $x$ or explicitly on time $t$, see [34], or when they are defined as linear operators acting on infinite dimensional spaces [27, 43].

The physical properties of $\mathrm{pH}$ systems are encoded in the algebraic structure of the coefficient matrices and in geometric structures associated with the flow of the differential equation. This leads to a remarkably robust modeling paradigm that greatly facilitates the combination and manipulation of $\mathrm{pH}$ systems. Note in particular that the family of $\mathrm{pH}$ systems is 
closed under power-conserving interconnection (see [28]); model reduction of $\mathrm{pH}$ systems via Galerkin projection yields (smaller) pH systems [2, 22, 39]; and conversely, $\mathrm{pH}$ systems are easily extendable in the sense that new state variables can be included while preserving the structure of (2). Thus, the range of application of the model can be increased while ensuring that basic conservation principles, as encoded in (4), remain in force.

When time-varying state constraints are included in a $\mathrm{pH}$ system, the resulting system is a port-Hamiltonian descriptor system (differential-algebraic equation) (pHDAE). Such pHDAE systems arise also in singularly perturbed $\mathrm{pH}$ systems when small parameters are set to zero, see [42]. Significantly, there is no systematic way that has yet emerged to describe this problem class consistently, in a way that reflects both the $\mathrm{pH}$ structure and the timevarying DAE structure appropriately from the point of view of DAE modeling. For implicit pH systems, implicit formulations have been studied in [19] relating to Dirac's work from 1950s [14]. However, we will allow general DAE system formulations and the first main topic of this paper is to propose such a systematic pHDAE approach. This is a challenging task, in particular when constraints of the DAE are 'hidden', which is often signaled with the terminology 'high-index DAE' [5, 30, 33]. Such DAEs are not well-suited for numerical simulation and control and so, either a reformulation or a regularization of the model must first be carried out, $[11,30]$. We will briefly summarize the fundamentals of this technique in Section 4.

Often Port-Hamiltonian DAEs are expected to be of differentiation-index at most one (see e.g., [42]). Such systems do not contain hidden constraints arising from derivatives. By way of contrast, we describe here a class of pHDAEs that arise from common modelling approaches that yield differentiation-indices higher than one, making regularization procedures necessary. Unfortunately, the usual regularization strategies do not preserve pHDAE model structure and so, the second main topic of this work paper is how one should accomplish regularization while respecting the pHDAE structure.

The paper is organized as follows. In Section 2 we give a definition of linear portHamiltonian differential-algebraic systems and demonstrate that this is a relevant class for many applications. The main properties of this new class of pHDAE systems (such as stability and dissipativity) are discussed in Section 3. Section 4 recalls the index reduction procedure for DAEs. The analysis of 'index at most one' pHDAEs is discussed in Section 5 while the structured regularization procedure for higher index systems is discussed in Section 6.

\section{Linear Port-Hamiltonian Differential-Algebraic Equations}

In this section we introduce a new definition of linear port-Hamiltonian descriptor systems (pHDAEs). As discussed in the introduction we would like to be able to combine the $\mathrm{pH}$ structure and the DAE structure in a systematic way. Our first observation to achieve this goal is that for a quadratic Hamiltonian $\mathcal{H}(x)=\frac{1}{2} x^{T} Q(t) x$ with $Q=Q^{T}$, the operator $\frac{d}{d t}-J(t) Q(t): \Omega \subset C^{1}\left(\mathbb{I}, \mathbb{R}^{n}\right) \rightarrow C^{0}\left(\mathbb{I}, \mathbb{R}^{n}\right)$ is skew-adjoint, so in order to extend this concept we first introduce linear skew-adjoint differential-algebraic operators, see [32] for the corresponding self-adjoint case.

Definition 1 A linear (differential-algebraic) operator

$$
\mathcal{L}:=\mathcal{E} \frac{d}{d t}-\mathcal{A}: \Omega \subset C^{1}\left(\mathbb{I}, \mathbb{R}^{n}\right) \rightarrow C^{0}\left(\mathbb{I}, \mathbb{R}^{n}\right)
$$


with coefficient functions $\mathcal{E} \in C^{1}\left(\mathbb{I}, \mathbb{R}^{n, n}\right), \mathcal{A} \in C^{0}\left(\mathbb{I}, \mathbb{R}^{n, n}\right)$ is called skew-adjoint, if $\mathcal{E}^{T}(t)=$ $\mathcal{E}(t)$ and $\dot{\mathcal{E}}(t)=-\left(\mathcal{A}(t)+\mathcal{A}^{T}(t)\right)$ for all $t \in \mathbb{I}$.

To further motivate this definition, observe that starting with vector functions $x_{1}(t), x_{2}(t)$ that are absolutely continuous on the interval $\mathbb{I}=\left(t_{0}, t_{f}\right)$ each with square integrable derivative and $x_{i}\left(t_{0}\right)=x_{i}\left(t_{f}\right)=0$ for $i=1,2$, and letting $\left\langle x_{1}, x_{2}\right\rangle=\int_{t_{0}}^{t_{f}} x_{2}^{T} x_{1} d t$ denote the usual $L_{2}$ inner product, we have

$$
\begin{aligned}
\left\langle x_{1}, \mathcal{L}\left(x_{2}\right)\right\rangle & =\left\langle x_{1}, \mathcal{E} \dot{x}_{2}-\mathcal{A} x_{2}\right\rangle=\left\langle x_{1}, \frac{d}{d t}\left(\mathcal{E} x_{2}\right)-\mathcal{A} x_{2}-\dot{\mathcal{E}} x_{2}\right\rangle \\
& =\left.x_{2}^{T} \mathcal{E}^{T} x_{1}\right|_{t_{0}} ^{t_{f}}-\left\langle\mathcal{E}^{T} \dot{x}_{1}, x_{2}\right\rangle-\left\langle\left(\mathcal{A}^{T}+\dot{\mathcal{E}}^{T}\right) x_{1}, x_{2}\right\rangle \\
& =\left\langle-\mathcal{E}^{T} \dot{x}_{1}-\left(\mathcal{A}^{T}+\dot{\mathcal{E}}^{T}\right) x_{1}, x_{2}\right\rangle=\left\langle-\mathcal{E} \dot{x}_{1}+\mathcal{A} x_{1}, x_{2}\right\rangle .
\end{aligned}
$$

So, the adjoint operator $\mathcal{L}^{*}$ formally satisfies $\mathcal{L}^{*}=-\mathcal{L}$. Note that boundary terms arising in partial integration will vanish under a wide variety of conditions that may replace the requirement of zero end conditions on $x_{1}(t)$ and $x_{2}(t)$.

Remark 2 In the context of densely-defined unbounded operators, recall that symmetric operators are those with adjoints that are extensions of the original operator, so one might use analogously the terminology skew-symmetric operator instead of skew-adjoint operator here. To be consistent with the terminology in [32] where self-adjoint DAE operators were introduced, we prefer to use its natural cousin, skew-adjoint operator.

One further motivation for introducing skew-adjoint operators in this way is that we would like to consider time-varying changes of basis and time-varying Galerkin projections. We show in the following result that linear skew-adjoint operators remain skew-adjoint under time-varying congruence transformations and Galerkin projections.

Lemma 3 Consider a linear skew-adjoint differential-algebraic operator

$$
\mathcal{L}:=\mathcal{E} \frac{d}{d t}-\mathcal{A}: \Omega \subset C^{1}\left(\mathbb{I}, \mathbb{R}^{n}\right) \rightarrow C^{0}\left(\mathbb{I}, \mathbb{R}^{n}\right)
$$

with coefficient functions $\mathcal{E} \in C^{1}\left(\mathbb{I}, \mathbb{R}^{n, n}\right)$ and $\mathcal{A} \in C^{0}\left(\mathbb{I}, \mathbb{R}^{n, n}\right)$. Then for every $\mathcal{V} \in C^{1}\left(\mathbb{I}, \mathbb{R}^{n, r}\right)$, the operator $\mathcal{L}_{\mathcal{V}}$ defined by

$$
\mathcal{L}_{\mathcal{V}}(x):=\mathcal{V}^{T} \mathcal{E} \mathcal{V} \dot{x}-\left(\mathcal{V}^{T} \mathcal{A} \mathcal{V}-\mathcal{V}^{T} \mathcal{E} \dot{\mathcal{V}}\right) x
$$

is again skew-adjoint, i.e., $\mathcal{L}_{\mathcal{V}}: \Omega \subset C^{1}\left(\mathbb{I}, \mathbb{R}^{r}\right) \rightarrow C^{0}\left(\mathbb{I}, \mathbb{R}^{r}\right)$ and $\mathcal{L}_{\mathcal{V}}^{*}=-\mathcal{L}_{\mathcal{V}}$.

Proof. Since $\mathcal{V}^{T} \mathcal{E} \mathcal{V}=\left(\mathcal{V}^{T} \mathcal{E} \mathcal{V}\right)^{T}$, it remains to consider the coefficient of $x$. Using $\mathcal{E}^{T}=\mathcal{E}$ and $\dot{\mathcal{E}}=-\left(\mathcal{A}+\mathcal{A}^{T}\right)$, we have

$$
\begin{aligned}
\frac{d}{d t}\left(\mathcal{V}^{T} \mathcal{E} \mathcal{V}\right) & =\dot{\mathcal{V}}^{T} \mathcal{E} \mathcal{V}+\mathcal{V}^{T} \dot{\mathcal{E}} \mathcal{V}+\mathcal{V}^{T} \mathcal{E} \dot{\mathcal{V}} \\
& =\dot{\mathcal{V}}^{T} \mathcal{E} \mathcal{V}-\mathcal{V}^{T}\left(\mathcal{A}+\mathcal{A}^{T}\right) \mathcal{V}+\mathcal{V}^{T} \mathcal{E} \dot{\mathcal{V}} \\
& =-\left(\mathcal{V}^{T} \mathcal{A} \mathcal{V}-\mathcal{V}^{T} \mathcal{E} \dot{\mathcal{V}}\right)-\left(\mathcal{V}^{T} \mathcal{A} \mathcal{V}-\mathcal{V}^{T} \mathcal{E} \dot{\mathcal{V}}\right)^{T}
\end{aligned}
$$

$\square$

Note that for any $t \in \mathbb{I}$ and $x \in C^{1}\left(\mathbb{I}, \mathbb{R}^{n}\right)$ we have $\mathcal{L}_{\mathcal{V}}(x(t))=\mathcal{V}^{T}(t) \mathcal{L}(\mathcal{V}(t) x(t))$. 
Remark 4 In Lemma $3, \mathcal{V}$ need be neither invertible nor square, and in particular a timevarying compression $\mathcal{V}=\left[\begin{array}{c}I_{r} \\ P(t)\end{array}\right]$ will produce a permissible skew-adjoint operator.

Skew-adjoint operators provide a natural extension of linear Hamilton operators with variable coefficients, so we may now incorporate dissipation terms and ports that leads us to a new definition of linear time-varying pHDAEs, which will be shown to be invariant under time-varying changes of basis. Our new definition somewhat extends related concepts discussed in [42].

Definition 5 A linear variable coefficient descriptor system of the form

$$
\begin{aligned}
E \dot{x} & =[(J-R) Q-E K] x+(B-P) u \\
y & =(B+P)^{T} Q x+(S+N) u,
\end{aligned}
$$

with $E, Q \in C^{1}\left(\mathbb{I}, \mathbb{R}^{n, n}\right), J, R, K \in C^{0}\left(\mathbb{I}, \mathbb{R}^{n, n}\right), B, P \in C^{0}\left(\mathbb{I}, \mathbb{R}^{n, m}\right), S=S^{T}, N=-N^{T} \in$ $C^{0}\left(\mathbb{I}, \mathbb{R}^{m, m}\right)$ is called port-Hamiltonian descriptor system (port-Hamiltonian differential-algebraic system) (pHDAE) if the following properties are satisfied:

(i) the differential-algebraic operator

$$
\mathcal{L}:=Q^{T} E \frac{d}{d t}-\left(Q^{T} J Q-Q^{T} E K\right): \mathcal{D} \subset C^{1}\left(\mathbb{I}, \mathbb{R}^{n}\right) \rightarrow C^{0}\left(\mathbb{I}, \mathbb{R}^{n}\right)
$$

is skew-adjoint, $i$. e. we have that $Q^{T} E \in C^{1}\left(\mathbb{I}, \mathbb{R}^{n, n}\right)$ and for all $t \in \mathbb{I}$,

$$
\begin{aligned}
Q^{T}(t) E(t) & =E^{T}(t) Q(t), \text { and } \\
\frac{d}{d t}\left(Q^{T}(t) E(t)\right) & =Q^{T}(t)[E(t) K(t)-J(t) Q(t)]+[E(t) K(t)-J(t) Q(t)]^{T} Q(t) ;
\end{aligned}
$$

(ii) the Hamiltonian function defined as

$$
\mathcal{H}(x):=\frac{1}{2} x^{T} Q^{T} E x: C^{1}\left(\mathbb{I}, \mathbb{R}^{n}\right) \rightarrow C^{1}(\mathbb{I}, \mathbb{R})
$$

is bounded from below by a constant, $\mathcal{H}(x(t)) \geq h_{0} \in \mathbb{R}$, uniformly for all $t \in \mathbb{I}$ and all solutions $x$ of (5);

(iii) the matrix function

$$
W:=\left[\begin{array}{cc}
Q^{T} R Q & Q^{T} P \\
P^{T} Q & S
\end{array}\right] \in C^{0}\left(\mathbb{I}, \mathbb{R}^{n+m, n+m}\right)
$$

is positive semidefinite, i. e., $W(t)=W^{T}(t) \geq 0$ for all $t \in \mathbb{I}$.

Note that in Definition 5 no further properties of the differential-algebraic operator are assumed. In particular it is not assumed that it has a certain index as a differential-algebraic equation.

Remark 6 The presence of the matrix function $K$ in (5) and subsequent expressions may be disconcerting, but note that a time-varying change of basis, $x=P(t) \tilde{x}$, will produce an additional term, $\dot{E} P$, in the transformed system that must be accommodated in order to retain the dissipation inequality. The matrix function $K(t)$ allows for this, and we include such a term from the beginning. 
Remark 7 Note that in the special case that $K(t)=0$ and $Q^{T} J Q$ is skew-symmetric, the skew-adjointness of $\mathcal{L}$ in $(6)$ implies that $E(t)^{T} Q(t)$ is constant in time, which is in accordance with the usual fact that for classical Hamiltonian systems $\dot{x}=J(x, t) \nabla_{x} \mathcal{H}(x)$ with quadratic Hamiltonian $\mathcal{H}(x)=\frac{1}{2} x^{T} Q x$ the matrix $Q$ is constant in time. The reason that we allow time-varying $E, Q$ is that these would arise when performing time-varying changes of basis, which will furthermore introduce a term $K \neq 0$ and make the coefficients time-varying.

Remark 8 Typically, $\mathrm{pH}$ systems are introduced via a Dirac structure $\mathcal{D}$, and for $(f, e) \in \mathcal{D}$ one has $e^{T} f+f^{T} e=0$ if there is no dissipation. Typically, linear dynamics is determined by choosing $e=Q x$, and $f=\dot{x}$. In the context of our new definition, then efforts and flows are defined as $e=Q x$ and $f=E \dot{x}$, respectively and, if there is not dissipation and $E^{T} Q$ is constant in time, then this would lead still to $\frac{d}{d t}\left(x^{T} E^{T} Q x\right)=e^{T} f+f^{T} e=0$. Notice that in contrast to the usual formulation, we allow for the possibility that both $E$ and $Q$ can be singular matrices.

Assumption (ii) in Definition 5 can be refined as follows:

Lemma 9 Assumption (ii) in Definition 5 is equivalent to the assertion that $\mathcal{H}(x(t)) \geq 0$ uniformly for all $t \in \mathbb{I}$ and all solutions $x$ of (5). In particular, the lower bound $h_{0}$ can be replaced by 0 .

Proof. One direction is obvious. For the other direction suppose that $\mathcal{H}(x)$ is semibounded for all solutions $x$ of (5), but say with $h_{0}<0$. Then there is $\hat{t} \in \mathbb{R}$ and a consistent initial condition $\hat{x}$ for $(5)$ at $t=\hat{t}$ with $u(t) \equiv 0$, such that $\mathcal{H}(\hat{x}(\hat{t}))<0$. By scaling $\hat{x}$ by $\kappa>\sqrt{\frac{h_{0}}{\mathcal{H}(\hat{x}(\hat{t}))}}$, $\kappa \hat{x}(\hat{t})$ is also a consistent initial condition for (5) at $t=\hat{t}$ with $u(t) \equiv 0$, and we find that $\mathcal{H}(\kappa \hat{x}(\hat{t}))=\kappa^{2} \mathcal{H}(\hat{x}(\hat{t}))<h_{0}$ giving a contradiction, so it must be that $h_{0} \geq 0$.

Lemma 9 shows that Assumption (ii) implies that $Q^{T} E$ is positive semidefinite on the solution set of (5), and so $Q(t)^{T} E(t) \geq 0$ for all $t \in \mathbb{I}$ is a sufficient condition for Assumption (ii) to hold.

We proceed to illustrate the generality of this new definition with some examples.

Example 10 Consider the model of a simple RLC network, see e. g. [13, 18], given by a linear constant coefficient DAE

$$
\underbrace{\left[\begin{array}{ccc}
G_{c} C G_{c}^{T} & 0 & 0 \\
0 & L & 0 \\
0 & 0 & 0
\end{array}\right]}_{:=E}\left[\begin{array}{c}
\dot{V} \\
\dot{I}_{l} \\
\dot{I}_{v}
\end{array}\right]=\underbrace{\left[\begin{array}{ccc}
-G_{r} R_{r}^{-1} G_{r}^{T} & -G_{l} & -G_{v} \\
G_{l}^{T} & 0 & 0 \\
G_{v}^{T} & 0 & 0
\end{array}\right]}_{:=(J-R) I}\left[\begin{array}{c}
V \\
I_{l} \\
I_{v}
\end{array}\right],
$$

with real symmetric constant matrices $L>0, C>0, R_{r}>0$ describing inductances, capacitances, and resistances, respectively that are present in the network. Here, $G_{v}$ is of full column rank, and the subscripts $r, c, l$, and $v$ refer to edge quantities corresponding to resistors, capacitors, inductors, and voltage sources. $V$ collects voltage drops across network branches, while $I$ collects the current fluxes through corresponding network branches. This model has a pHDAE structure with vanishing $B, P, S, N, K$; the matrix $Q$ is the identity; 
$E=E^{T}, J=-J^{T}, Q^{T} R Q=R \geq 0$, and

$$
\mathcal{H}=\left[\begin{array}{c}
V \\
I_{l} \\
I_{v}
\end{array}\right]^{T} E\left[\begin{array}{c}
V \\
I_{l} \\
I_{v}
\end{array}\right]=\left[\begin{array}{c}
V \\
I_{l}
\end{array}\right]^{T}\left[\begin{array}{cc}
G_{c} C G_{c}^{T} & 0 \\
0 & L
\end{array}\right]\left[\begin{array}{l}
V \\
I_{l}
\end{array}\right] .
$$

Note that the associated DAE has differentiation index 2, see [30].

Example 11 In $[15,16]$ the propagation of pressure waves on acoustic time scales through a network of gas pipelines is considered and an infinite-dimensional pHDAE is derived. A structure-preserving mixed finite element discretization leads to a block-structured constant coefficient pHDAE system

$$
\begin{aligned}
E \dot{x} & =(J-R) Q x+B u, \\
y & =B^{T} Q x, \\
x\left(t_{0}\right) & =x^{0},
\end{aligned}
$$

with $Q=I, P=0, S+N=0$,

$$
E=\left[\begin{array}{ccc}
M_{1} & 0 & 0 \\
0 & M_{2} & 0 \\
0 & 0 & 0
\end{array}\right], J=\left[\begin{array}{ccc}
0 & -\tilde{G} & 0 \\
\tilde{G}^{T} & 0 & \tilde{N}^{T} \\
0 & -\tilde{N} & 0
\end{array}\right], R=\left[\begin{array}{ccc}
0 & 0 & 0 \\
0 & \tilde{D} & 0 \\
0 & 0 & 0
\end{array}\right] B=\left[\begin{array}{c}
0 \\
\tilde{B}_{2} \\
0
\end{array}\right], x=\left[\begin{array}{c}
x_{1} \\
x_{2} \\
x_{3}
\end{array}\right],
$$

where the vector valued functions $x_{1}: \mathbb{R} \rightarrow \mathbb{R}^{n_{1}}, x_{2}: \mathbb{R} \rightarrow \mathbb{R}^{n_{2}}$ represent the discretized pressure and flux, respectively, and $x_{3}: \mathbb{R} \rightarrow \mathbb{R}^{n_{3}}$ represents the Lagrange multiplier for satisfying the space-discretized constraints. The coefficients $M_{1}=M_{1}^{T}, M_{2}=M_{2}^{T}$, and $\tilde{D}=\tilde{D}^{T}$ are positive definite, and the matrices $\tilde{N}$ and $\left[\begin{array}{ll}\tilde{G}^{T} & \tilde{N}^{T}\end{array}\right]^{T}$ have full row rank. The Hamiltonian is given by $\mathcal{H}(x)=\frac{1}{2} x^{T} E^{T} Q x=\frac{1}{2}\left(x_{1}^{T} M_{1} x_{1}+x_{2}^{T} M_{2} x_{2}\right)$.

The associated DAE in this case also has differentiation index 2, see [16, 30].

Definition 5 brings the $\mathrm{pH}$ modeling framework and the DAE framework together in a structured way. It should be noted, however, that in a DAE we may have hidden constraints that arise from differentiation, which are not explicitly formulated and the representation of the DAE that is used in simulation and control is not unique. One can for example add derivatives of constraints which leads to an over-determined system, then one can add dummy variables or Lagrange multipliers to make the number of variables equal to the number of equations or one can remove some of the dynamical equations to achieve this goal, see $[5,17,30,33]$ for detailed discussions on this topic. To rewrite these different formulations in the pHDAE formulation is not always obvious. Let us demonstrate this with an example from multi-body dynamics.

Example 12 A benchmark example for a DAE system is the model of a two-dimensional three-link mobile manipulator, see [6, 25], which after linearization around a stationary solution takes the form

$$
\begin{aligned}
M \ddot{p} & =-D \dot{p}-S p+G^{T} \lambda+B_{1} u, \\
0 & =-G p,
\end{aligned}
$$

with symmetric positive definite matrices $M, S$ and symmetric and positive semidefinite $D$. 
Besides the explicit constraint this system contains the first and second time derivative of $-G p=0$ as hidden algebraic constraints, see e. g. $[17,30]$. There are several regularization procedures that one can employ to make the system better suited for numerical simulation and control. One possibility is to replace the original constraint by its time derivative $0=-G \dot{p}$. In this case the model equation can easily be written in a pHDAE formulation. Adding a tracking output of the form $y=B_{1}^{T} \dot{p}$, see e. g., [26], and then transforming to first order form by introducing

$$
x=\left[\begin{array}{l}
x_{1} \\
x_{2} \\
x_{3}
\end{array}\right]:=\left[\begin{array}{c}
\dot{p} \\
p \\
\lambda
\end{array}\right],
$$

one obtains a linear pHDAE system $E \dot{x}=(J-R) Q x+B u, y=B^{T} Q x$, with

$$
\begin{aligned}
& E:= {\left[\begin{array}{ccc}
M & 0 & 0 \\
0 & I & 0 \\
0 & 0 & 0
\end{array}\right], R:=\left[\begin{array}{ccc}
D & 0 & 0 \\
0 & 0 & 0 \\
0 & 0 & 0
\end{array}\right], Q:=\left[\begin{array}{ccc}
I & 0 & 0 \\
0 & S & 0 \\
0 & 0 & I
\end{array}\right], } \\
& J:=\left[\begin{array}{ccc}
0 & -I & G^{T} \\
I & 0 & 0 \\
-G & 0 & 0
\end{array}\right], B:=\left[\begin{array}{c}
B_{1} \\
0 \\
0
\end{array}\right], P=0, S+N=0 .
\end{aligned}
$$

The Hamiltonian in this case is given by $\mathcal{H}(x)=\frac{1}{2}\left[\begin{array}{l}x_{1} \\ x_{2}\end{array}\right]^{T}\left[\begin{array}{cc}M & 0 \\ 0 & S\end{array}\right]\left[\begin{array}{l}x_{1} \\ x_{2}\end{array}\right]$.

Since the Lagrange multipliers in the multibody framework can be interpreted as external forces, it is also possible to incorporate them in the input $(B-P) u$ to achieve a pHDAE formulation as in Definition 5, but also other formulations are possible. For example, we may keep the original algebraic constraint as well and use an extra Lagrange multiplier for the first time derivative.

Besides explicit constraints, pHDAEs arise as a limiting situation in a singularly perturbed problem which has $\mathrm{pH}$ structure. Typical examples are mechanical multibody systems where small masses are ignored.

Example 13 Finite element modeling of the acoustic field in the interior of a car, see e. g. [36], leads to (after several simplifications) a large scale constant coefficient differentialalgebraic equation system of the form

$$
M \ddot{p}+D \dot{p}+K p=B_{1} u,
$$

where $p$ is the coefficient vector associated with the pressure in the air and the displacements of the structure, $B_{1} u$ is an external force, $M$ is a symmetric positive semidefinite mass matrix, $D$ is a symmetric positive semidefinite matrix, and $K$ is a symmetric positive definite stiffness matrix. Here $M$ is only semidefinite, since small masses were set to zero, so $M$ is a perturbation of a positive definite matrix. The resulting first-order formulation yields the state equation of a pHDAE system, $E \dot{z}=(J-R) Q z+B u$, where

$$
\begin{aligned}
E & :=\left[\begin{array}{cc}
M & 0 \\
0 & I
\end{array}\right], J:=\left[\begin{array}{cc}
0 & -I \\
I & 0
\end{array}\right], R:=\left[\begin{array}{cc}
D & 0 \\
0 & 0
\end{array}\right], z:=\left[\begin{array}{c}
\dot{p} \\
p
\end{array}\right], \\
Q & :=\left[\begin{array}{cc}
I & 0 \\
0 & K
\end{array}\right], B:=\left[\begin{array}{c}
B_{1} \\
0
\end{array}\right], P:=0,
\end{aligned}
$$


and the Hamiltonian is

$$
\mathcal{H}=\frac{1}{2}\left(z^{T} E^{T} Q z\right)=\frac{1}{2}\left(\dot{p}^{T} M \dot{p}+p^{T} K p\right) .
$$

Note that this model is nonlinear originally, but simplifications carried out in the modeling process, e. g. linearization and omission of nonlinear terms with small coefficients, lead to a linear model. In this example, as long as $M$ is invertible the implicit $\mathrm{pH}$ formulation of [19] could be employed to transform the system to a usual $\mathrm{pH}$ system, however, this approach would not be possible in the limiting situation and in the context of numerical methods, the small masses problem behaves just as the singular case.

Remark 14 A special case of (5) takes the following form:

$$
\begin{aligned}
E \dot{x} & =(J-R) x+(B-P) u, \\
y & =(B+P)^{T} x+(S+N) u,
\end{aligned}
$$

where $E=E^{T} \in C^{1}\left(\mathbb{I}, \mathbb{R}^{n, n}\right), R=R^{T}, J \in C^{0}\left(\mathbb{I}, \mathbb{R}^{n, n}\right), B, P \in C^{0}\left(\mathbb{I}, \mathbb{R}^{n, m}\right), S=S^{T}, N=$ $-N^{T} \in C^{0}\left(\mathbb{I}, \mathbb{R}^{m, m}\right)$ as before but now we require that

(i) the differential algebraic operator

$$
\mathcal{L}:=E \frac{d}{d t}-J: \mathcal{D} \subset C^{1}\left(\mathbb{I}, \mathbb{R}^{n}\right) \rightarrow C^{0}\left(\mathbb{I}, \mathbb{R}^{n}\right)
$$

is skew-adjoint, so that we have for all $t \in \mathbb{I}$,

$$
\frac{d}{d t} E(t)=-\left[J(t)+J(t)^{T}\right] ;
$$

(ii) $E(t)$ is bounded from below by a constant symmetric matrix $E_{0}$.

(iii) $W(t):=\left[\begin{array}{ll}R(t) & P(t) \\ P^{T}(t) & S(t)\end{array}\right] \geq 0$ for all $t \in \mathbb{I}$.

The effective Hamiltonian is now

$$
\mathcal{H}(x):=\frac{1}{2} x^{T} E x: C^{1}\left(\mathbb{I}, \mathbb{R}^{n}\right) \rightarrow \mathbb{R} .
$$

Notice that in this model description we have merged the roles of $Q$ and $E$. This is always possible when $Q$ is pointwise invertible, see Section 3, but this formulation may not be possible when $Q$ is singular, see [35].

Our new definition of pHDAEs allows for somewhat greater flexibility in modeling portHamiltonian systems with constraints. The non-uniqueness in the factorization of the Hessian $E^{T} Q$ of the Hamiltonian allows one to incorporate singularities which may otherwise be hard to deal with. In the next section we will show that the classical properties of $\mathrm{pH}$ systems are retained and that the $\mathrm{pH}$ structure remains invariant under time-varying transformations. 


\section{Properties of pHDAE systems}

To analyze the properties of pHDAE systems, we first derive the dissipation inequality.

Theorem 15 Consider the linear time-varying system (5) and assume that this system satisfies condition i) of Definition 5. Then its (classical) solutions satisfy

$$
\frac{d}{d t} \mathcal{H}(x)=u^{T} y-\left[\begin{array}{l}
x \\
u
\end{array}\right]^{T} W\left[\begin{array}{l}
x \\
u
\end{array}\right],
$$

where $W$ is defined in (8).

Furthermore we have the following properties.

i) If $W \equiv 0$, then $\frac{d}{d t} \mathcal{H}=u^{T} y$.

ii) If $W \geq 0$ for all $t \in \mathbb{I}$, then the system satisfies the dissipation inequality (4).

Proof. By Definition 5 we have

$$
\begin{aligned}
\frac{d}{d t} \mathcal{H}= & \frac{1}{2}\left[\dot{x}^{T}\left(Q^{T} E\right) x+x^{T} \frac{d}{d t}\left(Q^{T} E\right) x+x^{T}\left(Q^{T} E\right) \dot{x}\right] \\
= & \frac{1}{2} x^{T} \frac{d}{d t}\left(Q^{T} E\right) x+x^{T} Q^{T}(E \dot{x}) \\
= & \frac{1}{2} x^{T} \frac{d}{d t}\left(Q^{T} E\right) x+x^{T} Q^{T}([J Q-R Q-E K] x+B u-P u) \\
= & \frac{1}{2} x^{T} \frac{d}{d t}\left(Q^{T} E\right) x+x^{T} Q^{T} J Q x-x^{T} Q^{T} R Q x-x^{T} Q^{T} E K x-x^{T} Q^{T} P u+u^{T} B^{T} Q x \\
= & \frac{1}{2} x^{T} \frac{d}{d t}\left(Q^{T} E\right) x+x^{T} Q^{T} J Q x-x^{T} Q^{T} R Q x-x^{T} Q^{T} E K x-x^{T} Q^{T} P u
\end{aligned}
$$

From the skew-adjointness of $\mathcal{L}$ we then have that

$$
\frac{d}{d t} \mathcal{H}=u^{T} y-\left[\begin{array}{l}
x \\
u
\end{array}\right]^{T} W\left[\begin{array}{l}
x \\
u
\end{array}\right] .
$$

Part i) then follows immediately from the assumption $W \equiv 0$, while in Part ii) the fact that $W(t) \geq 0$ for all $t \in \mathbb{I}$ implies that for any $t_{1} \geq t_{0}$,

$$
\mathcal{H}\left(x\left(t_{1}\right)\right)-\mathcal{H}\left(x\left(t_{0}\right)\right)=\int_{t_{0}}^{t_{1}} \frac{d}{d t} \mathcal{H} d t \leq \int_{t_{0}}^{t_{1}} y^{T} u d t .
$$


Remark 16 Theorem 15 connects structural features of pHDAEs with the assertion that $u^{T} y$ bounds the instantaneous rate of change of the Hamiltonian function $\mathcal{H}(x)$, implying in turn that the dissipation inequality (4) holds. This is a conservation principle that must hold notwithstanding particular interpretations of $\mathcal{H}(x)$ as system "energy" (an interpretation which might otherwise require further qualification if there is time variation in $\left.E(t)^{T} Q(t)\right)$. Indeed, the strength of this new formulation is that it allows retention of a general conservation principle even in the face of time-varying changes of basis, which arise naturally in considering nonlinear systems that are linearized along nonstationary solutions, when the solution manifold or constraints are moving in time.

Remark 17 It follows from Theorem 15 with the formulation of flows and efforts as in Remark 8 that if $W=0$, then we have the usual relationship for Hamiltonian systems that $e^{T} f+y^{T} u=0$.

Another important feature of our definition of pHDAE systems is that a change of basis and a scaling with an invertible matrix function preserves the pHDAE structure and the Hamiltonian.

Theorem 18 Consider a pHDAE system of the form (5) with Hamiltonian (7). Let $U \in$ $C^{0}\left(\mathbb{I}, \mathbb{R}^{n, n}\right)$ and $V \in C^{1}\left(\mathbb{I}, \mathbb{R}^{n, n}\right)$ be pointwise invertible in $\mathbb{I}$. Then the transformed DAE

$$
\begin{aligned}
\tilde{E} \dot{\tilde{x}} & =[(\tilde{J}-\tilde{R}) \tilde{Q}-\tilde{E} \tilde{K}] \tilde{x}+(\tilde{B}-\tilde{P}) u \\
y & =(\tilde{B}+\tilde{P})^{T} \tilde{Q} \tilde{x}+(S+N) u
\end{aligned}
$$

with

$$
\begin{aligned}
\tilde{E} & =U^{T} E V, \quad \tilde{Q}=U^{-1} Q V, & & \tilde{J}=U^{T} J U, \\
\tilde{R} & =U^{T} R U, \quad \tilde{B}=U^{T} B, & & \tilde{P}=U^{T} P, \\
\tilde{K} & =V^{-1} K V+V^{-1} \dot{V}, & & x=V \tilde{x}
\end{aligned}
$$

is still a pHDAE system with the equivalent Hamiltonian $\tilde{\mathcal{H}}(\tilde{x})=\frac{1}{2} \tilde{x}^{T} \tilde{Q}^{T} \tilde{E} \tilde{x}=\mathcal{H}(x)$.

Proof. The transformed DAE system is obtained from the original DAE system by setting $x=V \tilde{x}$ in (5), by pre-multiplying with $U^{T}$, and by inserting $U U^{-1}$ in front of $Q$. The transformed operator corresponding to $\mathcal{L}$ in $(6)$ is

$$
\mathcal{L}_{V}:=\tilde{Q}^{T} \tilde{E} \frac{d}{d t}-\tilde{Q}^{T}(\tilde{J} \tilde{Q}-\tilde{E} \tilde{K})
$$

Because

$$
\tilde{Q}^{T} \tilde{E}=V^{T} Q^{T} E V, \quad \tilde{Q}^{T} \tilde{J} \tilde{Q}=V^{T} Q^{T} J Q V, \quad \tilde{Q}^{T} \tilde{E} V^{-1} \dot{V}=V^{T} Q^{T} E \dot{V},
$$

by Lemma $3, \mathcal{L}_{V}$ is skew-adjoint, since $\mathcal{L}$ defined in (6) is skew-adjoint. Hence,

$$
\begin{aligned}
\tilde{Q}^{T} \tilde{E} & =\tilde{E}^{T} \tilde{Q} \\
\frac{d}{d t}\left(\tilde{Q}^{T} \tilde{E}\right) & =-\tilde{Q}^{T}(\tilde{J} \tilde{Q}-\tilde{E} \tilde{K})-(\tilde{J} \tilde{Q}-\tilde{E} \tilde{K})^{T} \tilde{Q} .
\end{aligned}
$$


It is straightforward to show that $\tilde{\mathcal{H}}(\tilde{x})=\mathcal{H}(x)$ and

$$
\frac{d}{d t} \tilde{\mathcal{H}}(\tilde{x})=y^{T} u-\left[\begin{array}{l}
\tilde{x} \\
u
\end{array}\right]^{T} \tilde{W}\left[\begin{array}{l}
\tilde{x} \\
u
\end{array}\right]
$$

where

$$
\begin{aligned}
\tilde{W} & =\left[\begin{array}{cc}
\tilde{Q}^{T} \tilde{R} \tilde{Q} & \tilde{Q}^{T} \tilde{P} \\
\tilde{P}^{T} \tilde{Q} & S
\end{array}\right]=\left[\begin{array}{cc}
V^{T} Q^{T} R Q V & V^{T} Q^{T} P \\
P^{T} Q V & S
\end{array}\right] \\
& =\left[\begin{array}{ll}
V & 0 \\
0 & I
\end{array}\right]^{T} W\left[\begin{array}{ll}
V & 0 \\
0 & I
\end{array}\right],
\end{aligned}
$$

and $W$ is defined in (8). Because $W(t)$ is positive semidefinite for all $t \in \mathbb{I}$, so is $\tilde{W}(t)$. Therefore, for any $t_{1} \geq t_{0}$,

$$
\tilde{\mathcal{H}}\left(\tilde{x}\left(t_{1}\right)\right)-\tilde{\mathcal{H}}\left(\tilde{x}\left(t_{0}\right)\right) \leq \int_{t_{0}}^{t_{1}} y^{T}(t) u(t) d t,
$$

which establishes the dissipation inequality.

An important point to note is that the Hamiltonian stays invariant under time-varying changes of basis and the operator $\mathcal{L}_{V}$, the Hamiltonian $\tilde{\mathcal{H}}(\tilde{x})$, and the matrix function $\tilde{W}$ are independent of the choice of the matrix function $U$.

As we have already pointed out, our definition of pHDAE systems has the extra term $-E K x$ on the right hand side which is needed to incorporate time-varying changes of basis. Even if $K=0$ in the original system, after the transformation given in Theorem 18 the extra term $-\tilde{E} \tilde{K}$ with $\tilde{K}=V^{-1} \dot{V}$ will appear. Note that if an orthogonal change of basis is carried out in a system with $K=0$ then the resulting $\tilde{K}=V^{-1} \dot{V}$ is skew-symmetric. However, even if $K \neq 0$, this term can be removed via a change of basis transformation which does not change the Hamiltonian.

Lemma 19 Consider a pHDAE system

$$
\begin{aligned}
\tilde{E} \dot{\tilde{x}} & =[(\tilde{J}-\tilde{R}) \tilde{Q}-\tilde{E} \tilde{K})] \tilde{x}+(\tilde{B}-\tilde{P}) u \\
y & =(\tilde{B}+\tilde{P})^{T} \tilde{Q} \tilde{x}+(S+N) u
\end{aligned}
$$

with Hamiltonian $\tilde{\mathcal{H}}(\tilde{x})=\frac{1}{2} \tilde{x}^{T} \tilde{Q}^{T} \tilde{E} \tilde{x}$, where $\tilde{K} \in C\left(\mathbb{I}, \mathbb{R}^{n, n}\right)$. If $V_{\tilde{K}} \in C^{1}\left(\mathbb{I}, \mathbb{R}^{n, n}\right)$ is a pointwise invertible solution of the matrix differential equation $\dot{V}=V \tilde{K}$ (e. g. with the initial condition $\left.V\left(t_{0}\right)=I\right)$, then defining

$$
\begin{aligned}
E & =\tilde{E} V_{K}^{-1}, & & Q \tilde{Q} V_{k}^{-1}, \\
J & =\tilde{J}, & & R=\tilde{R}, \quad B=\tilde{B}, \\
P & =\tilde{P}, & & \tilde{x}=V_{K}^{-1} x,
\end{aligned}
$$

the system

$$
\begin{aligned}
E \dot{x} & =(J-R) Q x+(B-P) u \\
y & =(B+P)^{T} Q x+(S+N) u
\end{aligned}
$$

is again $p H D A E$ with the equivalent Hamiltonian $\mathcal{H}(x)=\tilde{\mathcal{H}}(\tilde{x})=\frac{1}{2} x^{T} Q^{T} E x$. 
Proof. For a given matrix function $\tilde{K}$, the system $\dot{V}=V \tilde{K}$ always has a solution $V_{K}$ that is pointwise invertible. The remainder of the proof follows by reversing the proof of Theorem 18 with $U=I$ and using that $\dot{V}_{K} V_{K}^{-1}=-V_{K} \frac{d}{d t}\left(V_{K}^{-1}\right)$.

Note again that if $K$ is real and skew-symmetric, then the matrix function $V_{K}$ in Lemma 19 can be chosen to be pointwise real orthogonal.

Remark 20 Following Theorem 18, if $E$ is pointwise invertible, then the original system can be transformed into one with $\hat{E}=I$, and so, into a standard port-Hamiltonian system. Whenever $Q$ is pointwise invertible, then the original system can be transformed into the one with new $\hat{Q}=I$, see Remark 14. Which of these formulations is preferable will depend on the sensitivity (conditioning) of these transformations. In the context of numerical simulation and control methods, these transformations should be avoided if they are ill-conditioned. The representation with $E$ and $Q$ also has the advantage that it is more robust to perturbations as has been shown recently for the constant coefficient case in [20] in the context of computing stability distances and that it leads to structured canonical and condensed forms $[35,46]$.

\section{Regularization of DAEs}

To study the DAE properties of pHDAES, in this section we briefly recall the index reduction and reformulation procedure for DAE systems and then modify these results to pHDAEs. We follow the general procedure derived in detail in [30] and rewrite our system as a general descriptor system of the form

$$
\begin{aligned}
F(t, x, \dot{x}, u) & :=E \dot{x}-A x-B u=0 \\
x\left(t_{0}\right) & =x^{0} \\
y & =G(t, x, u):=C x+D u
\end{aligned}
$$

Note that here, in contrast to the more general case in [11], we assume square systems with an equal number of equations and variables and with an equal number of inputs and outputs.

For the analysis and regularization procedure we make use of the behavioral approach [38], which introduces a descriptor vector $v=\left[x^{T}, u^{T}\right]^{T}$, and the behavioral formulation

$$
\mathcal{F}(t, v, \dot{v})=0,
$$

together with a set of initial conditions $c\left(v\left(t_{0}\right)\right)=v^{0}$ which results from the original initial condition. Then one forms a derivative array, see [9],

$$
\mathcal{F}_{\mu}\left(t, v, \dot{v}, \ldots, v^{(\mu+1)}\right)=0,
$$

stacking the equation and its time derivatives up to level $\mu$ into one large system. We denote partial derivatives of $\mathcal{F}_{\mu}$ with respect to selected variables $\zeta$ from $v_{\mu}:=\left(t, v, \dot{v}, \ldots, v^{(\mu+1)}\right)$ by $\mathcal{F}_{\mu ; \zeta}$, and the solution set of the algebraic equation associated with the derivative array $\mathcal{F}_{\mu}$ for some integer $\mu$ (considering variables as well as their derivatives as algebraic variables) by $\mathbb{L}_{\mu}$.

The main assumption for the analysis is that the DAE satisfies the following hypothesis, which in the linear case under some constant rank assumptions can be proved as a theorem, see [30]. 
Hypothesis 21 Consider the system of nonlinear DAEs (17). There exist integers $\mu, r, a$, $d$, and $\nu$ such that $\mathbb{L}_{\mu}$ is not empty and such that for every $v_{\mu}^{0}=\left(t_{0}, v_{0}, \dot{v}_{0}, \ldots, v_{0}^{(\mu+1)}\right) \in \mathbb{L}_{\mu}$ there exists a neighborhood in which the following properties hold.

1. The set $\mathbb{L}_{\mu} \subseteq \mathbb{R}^{(\mu+2)(n+m)+1}$ forms a manifold of dimension $(\mu+2)(n+m)+1-r$.

2. We have $\operatorname{rank} \mathcal{F}_{\mu ; v, \dot{v}, \ldots, v^{(\mu+1)}}=r$ on $\mathbb{L}_{\mu}$.

3. We have corank $\mathcal{F}_{\mu ; v, \dot{v}, \ldots, v^{(\mu+1)}}-\operatorname{corank} \mathcal{F}_{\mu-1 ; v, \dot{v}, \ldots, v^{(\mu)}}=\nu$ on $\mathbb{L}_{\mu}$, where the corank is the dimension of the corange and the convention is used that corank of $\mathcal{F}_{-1 ; v}$ is 0 .

4. We have $\operatorname{rank} \mathcal{F}_{\mu ; \dot{v}, \ldots, v(\mu+1)}=r-a$ on $\mathbb{L}_{\mu}$ such that there exist smooth full rank matrix functions $Z_{2}$ and $T_{2}$ of size $(\mu+1) n \times a$ and $(n+m) \times(n+m-a)$, respectively, satisfying $Z_{2}^{T} \mathcal{F}_{\mu ; \dot{v}, \ldots, v^{(\mu+1)}}=0$, rank $Z_{2}^{T} \mathcal{F}_{\mu ; v}=a$, and $Z_{2}^{T} \mathcal{F}_{\mu ; v} T_{2}=0$ on $\mathbb{L}_{\mu}$.

5. We have $\operatorname{rank} \mathcal{F}_{\dot{v}} T_{2}=d=n-a-\nu$ on $\mathbb{L}_{\mu}$ such that there exists a smooth full rank matrix function $Z_{1}$ of size $n \times d$ satisfying $\operatorname{rank} Z_{1}^{T} \mathcal{F}_{\dot{v}} T_{2}=d$.

The smallest $\mu$ for which Hypothesis 21 holds is called the strangeness-index of (17), see [30]. It generalizes the concept of differentiation-index [5] to over- and under-determined systems but in contrast to the differentiation-index, ordinary differential equations and purely algebraic equations have $\mu=0$ and for other systems the differentiation-index (if defined) is $\mu+1$, see [30]. The quantity $\nu$ gives the number of trivial equations $0=0$ in the system. Of course, these equations can be simply removed and so for our further analysis we assume that $\nu=0$.

If Hypothesis 21 holds then, in the original variables $x$ and $u$ locally (via the implicit function theorem) there exists, see [29,30], a reformulation of the system (in the same variables) and a partitioning of the projection matrix $Z_{2}$ into two parts, so that the system takes the form

$$
\begin{aligned}
\hat{\mathcal{F}}_{1}(t, x, \dot{x}, u) & =0, \\
\hat{\mathcal{F}}_{2}(t, x, u) & =0, \\
\hat{\mathcal{F}}_{3}(t, x) & =0,
\end{aligned}
$$

in which the first set of $d$ equations (with $\hat{\mathcal{F}}_{1}=Z_{1}^{T} \mathcal{F}$ ) form a (linear) projection of the original set of equations representing the dynamics of the system, while the second and third sets of equations contain all explicit and hidden algebraic constraints that can be used to parameterize the solution manifold and to characterize when an initial condition is consistent.

It should also be noted that although formally also derivatives of $u$ have been used to form the derivative array, no derivatives of $u$ appear in the regularized system (19). This has been shown in various contexts $[7,30,31]$ and is due to the fact that only derivatives of algebraic equations that cannot be influenced by the control (non-impulse controllable equations) are needed to generate (19) leading to the third equation of (19).

System (19) has the property that it can be made to be of differentiation index at most one by an appropriate feedback.

As mentioned in the introduction, it is a common expectation that port-Hamiltonian DAEs will have a differentiation-index at most one (i.e., they satisfy Hypothesis 21 with $\mu=0$ ). Example 10 gives a typical illustration of a system that violates this expectation, in this case 
modeling a simple electrical circuit. Indeed, in Example 10 we have $Z_{2}^{T}=\left[\begin{array}{lll}0 & 0 & I\end{array}\right]$ and obtain

$$
\bar{E}=\left[\begin{array}{ccc}
G_{c} C G_{c}^{T} & 0 & 0 \\
0 & L & 0 \\
-G_{v}^{T} & 0 & 0
\end{array}\right]
$$

which is clearly not invertible, except if the last row and column is empty. The same matrix $Z_{2}$ can be used in Example 11 and yields

$$
\bar{E}=\left[\begin{array}{ccc}
M_{1} & 0 & 0 \\
0 & M_{2} & 0 \\
0 & \tilde{N} & 0
\end{array}\right]
$$

which is also not invertible except if the last row and column is empty. Due to this special structure both systems have $\mu=1$, i. e., both have differentiation-index two, when the input is chosen to be 0 . The analysis of Example 12 with the original constraint $0=-G p$ has $\mu=2$ (differentiation-index three) and the formulation as pHDAE in Example 12 has $\mu=1$ (differentiation-index two) if $G G^{T}$ is invertible, see e. g. [5, 30].

Adding the output equations to the system, in the linear time varying case (and also locally in the nonlinear case), we obtain a system of the form

$$
\begin{aligned}
\hat{E}_{1} \dot{x} & =\hat{A}_{1} x+B_{1} u, \\
0 & =\hat{A}_{2} x+B_{2} u, \\
0 & =\hat{A}_{3} x, \\
x\left(t_{0}\right) & =x^{0} \\
y & =C x+D u .
\end{aligned}
$$

Note that the first two equations in (20) can be obtained directly from the original system and, as stated before, they contain the ordinary differential equations as well as the equations for which one can find an initial feedback $u=\mathcal{K} x+\tilde{u}$ so that the matrix function

$$
\left[\begin{array}{c}
\hat{E}_{1} \\
\hat{A}_{2}+B_{2} \mathcal{K} \\
\hat{A}_{3}
\end{array}\right]
$$

is pointwise invertible; the resulting system is strangeness-free (of differentiation-index one) considered as a system with input $\tilde{u}=0$, see $[3,11]$ for a detailed analysis and regularization procedures. In the following we assume that this reinterpretation has been done, see [30].

Furthermore, there exists a partitioning of the variables so that the first three equations in (20) take the form

$$
\left[\begin{array}{ccc}
\hat{E}_{11} & \hat{E}_{12} & \hat{E}_{13} \\
0 & 0 & 0 \\
0 & 0 & 0
\end{array}\right]\left[\begin{array}{c}
\dot{x}_{1} \\
\dot{x}_{2} \\
\dot{x}_{3}
\end{array}\right]=\left[\begin{array}{ccc}
\hat{A}_{11} & \hat{A}_{12} & \hat{A}_{13} \\
\hat{A}_{21} & \hat{A}_{22} & \hat{A}_{23} \\
\hat{A}_{31} & \hat{A}_{32} & \hat{A}_{33}
\end{array}\right]\left[\begin{array}{c}
x_{1} \\
x_{2} \\
x_{3}
\end{array}\right]+\left[\begin{array}{c}
\hat{B}_{1} \\
\hat{B}_{2} \\
0
\end{array}\right] u
$$

with the special property that $\hat{A}_{33}$ is invertible and the reduced system obtained by solving for $x_{3}$ is strangeness-free (of differentiation index at most one) when setting $u=0$.

The regularization procedure described here holds for general DAEs but it does not reflect or maintain the underlying port-Hamiltonian structure, so in the next two sections we modify this approach for systems with a pHDAE structure to rectify this shortcoming. 


\section{PHDAEs of differentiation-index at most one}

In this section we characterize linear time-varying pHDAE systems of differentiation-index at most one $(\mu=0)$. In this case Hypothesis 21 implies that the matrix function $E(t)$ has constant rank. Then, see e. g., Theorem 3.9 in [30], there exist pointwise orthogonal matrix functions $\tilde{U}$ and $\tilde{V}$ such that

$$
\tilde{U}^{T} E \tilde{V}=\left[\begin{array}{cc}
E_{11} & 0 \\
0 & 0
\end{array}\right]=: \tilde{E}
$$

where $E_{11}$ is pointwise invertible. Because $Q^{T} E$ is real symmetric, setting

$$
\tilde{U}^{T} Q \tilde{V}=\left[\begin{array}{ll}
Q_{11} & Q_{12} \\
Q_{21} & Q_{22}
\end{array}\right]
$$

one has $Q_{11}^{T} E_{11}=E_{11}^{T} Q_{11}$ and also $Q_{12}=0$. Partition in the same way

$$
\begin{aligned}
\tilde{U}^{T} J \tilde{U} & =\left[\begin{array}{ll}
\tilde{J}_{11} & \tilde{J}_{12} \\
\tilde{J}_{21} & J_{22}
\end{array}\right], \quad \tilde{U}^{T} R \tilde{U}=\left[\begin{array}{ll}
\tilde{R}_{11} & \tilde{R}_{12} \\
\tilde{R}_{12}^{T} & R_{22}
\end{array}\right], \\
\tilde{U}^{T}(J-R) \tilde{U} & =\left[\begin{array}{ll}
\tilde{J}_{11} & \tilde{J}_{12} \\
\tilde{J}_{21} & J_{22}
\end{array}\right]-\left[\begin{array}{ll}
\tilde{R}_{11} & \tilde{R}_{12} \\
\tilde{R}_{12}^{T} & R_{22}
\end{array}\right]=:\left[\begin{array}{ll}
\tilde{L}_{11} & \tilde{L}_{12} \\
\tilde{L}_{21} & L_{22}
\end{array}\right], \\
\tilde{K} & =\tilde{V}^{T}(K \tilde{V}+\dot{\tilde{V}})=\left[\begin{array}{cc}
\tilde{K}_{11} & K_{12} \\
\tilde{K}_{21} & K_{22}
\end{array}\right] .
\end{aligned}
$$

Since the system has differentiation-index at most one, the block $L_{22} Q_{22}$ either does not occur (in this case we have an implicitly defined standard $\mathrm{pH}$ system) or it must be pointwise invertible, see [30], i. e., both $L_{22}$ and $Q_{22}$ are pointwise invertible. Let $U=\tilde{U} T$, where

$$
T:=\left[\begin{array}{cc}
I & 0 \\
T_{21} & I
\end{array}\right], \quad T_{21}=-L_{22}^{-T}\left(\tilde{L}_{12}-E_{11} K_{12} Q_{22}^{-1}\right)^{T} .
$$

Then a transformation of the original pHDAE with $U$ and $\tilde{V}$ yields a transformed pHDAE system, where $\tilde{K}$ is defined above,

$$
\begin{aligned}
\tilde{E} & =U^{T} E \tilde{V}=\tilde{U}^{T} E \tilde{V}, \quad \tilde{Q}=U^{-1} Q \tilde{V}=\left[\begin{array}{ll}
Q_{11} & 0 \\
\tilde{Q}_{21} & Q_{22}
\end{array}\right], \quad \tilde{S}=S, \quad \tilde{N}=N, \\
\tilde{J} & =U^{T} J U=\left[\begin{array}{ll}
J_{11} & J_{12} \\
J_{21} & J_{22}
\end{array}\right], \quad \tilde{R}=U^{T} R U=\left[\begin{array}{ll}
R_{11} & R_{12} \\
R_{12}^{T} & R_{22}
\end{array}\right], \\
\tilde{L} & =\tilde{J}-\tilde{R}=\left[\begin{array}{ll}
J_{11} & J_{12} \\
J_{21} & J_{22}
\end{array}\right]-\left[\begin{array}{ll}
R_{11} & R_{12} \\
R_{12}^{T} & R_{22}
\end{array}\right]=\left[\begin{array}{ll}
L_{11} & L_{12} \\
L_{21} & L_{22}
\end{array}\right],
\end{aligned}
$$

and

$$
\tilde{L} \tilde{Q}-\tilde{E} \tilde{K}=\left[\begin{array}{cc}
L_{11} Q_{11}+L_{12} \tilde{Q}_{21}-E_{11} \tilde{K}_{11} & 0 \\
L_{21} Q_{11}+L_{22} \tilde{Q}_{21} & L_{22} Q_{22}
\end{array}\right]
$$

That is,

$$
\left(J_{12}-R_{12}\right) Q_{22}-E_{11} K_{12}=0 .
$$

Performing another change of basis to make $\tilde{Q}$ (block) diagonal with a transformation matrix

$$
\tilde{T}:=\left[\begin{array}{cc}
I & 0 \\
-Q_{22}^{-1} \tilde{Q}_{21} & I
\end{array}\right]
$$


then setting $V=\tilde{V} \tilde{T}$ and transforming the original system with $U, V$ we obtain that any pHDAE of differentiation-index one can be transformed to the form

$$
\begin{aligned}
{\left[\begin{array}{cc}
E_{11} & 0 \\
0 & 0
\end{array}\right]\left[\begin{array}{l}
\dot{x}_{1} \\
\dot{x}_{2}
\end{array}\right]=} & \left(\left[\begin{array}{cc}
L_{11} & L_{12} \\
L_{21} & L_{22}
\end{array}\right]\left[\begin{array}{cc}
Q_{11} & 0 \\
0 & Q_{22}
\end{array}\right]-\left[\begin{array}{cc}
E_{11} K_{11} & E_{11} K_{12} \\
0 & 0
\end{array}\right]\right)\left[\begin{array}{l}
x_{1} \\
x_{2}
\end{array}\right] \\
& +\left[\begin{array}{l}
B_{1}-P_{1} \\
B_{2}-P_{2}
\end{array}\right] u \\
y & =\left[\left(B_{1}+P_{1}\right)^{T}\left(B_{2}+P_{2}\right)^{T}\right]\left[\begin{array}{cc}
Q_{11} & 0 \\
0 & Q_{22}
\end{array}\right]\left[\begin{array}{l}
x_{1} \\
x_{2}
\end{array}\right]+(S+N) u
\end{aligned}
$$

where (22) holds, with

$$
K_{11}=\tilde{K}_{11}-K_{12} Q_{22}^{-1} \tilde{Q}_{21}^{-1}, \quad\left[\begin{array}{cc}
B_{1} & P_{1} \\
B_{2} & P_{2}
\end{array}\right]=U^{T}\left[\begin{array}{ll}
B & P
\end{array}\right] .
$$

Following Theorem 18 this transformation will not change the Hamiltonian, and (23) is still a pHDAE of index at most one. Note that these transformations should not be performed in a numerical integration or control design technique, since the inversion of the matrices $Q_{22}$ and $L_{22}$ may be highly ill-conditioned. However, from an analytic point of view we have the following theorem.

Theorem 22 Suppose that the pHDAE system (5) is of differentiation-index at most one (i.e., it satisfies Hypothesis 21 with $\mu=0$ ) that $\nu=0$, and that $E(t)$ has constant rank. Assume further that the system is transformed to the form (22)-(23). Then for any input function $u$ and $x_{1}\left(t_{0}\right)=x_{1,0}$ the first component of the solution and the output of (23) are given by reduced implicit pHDAE system

$$
\begin{aligned}
E_{11} \dot{x}_{1} & =\left[\left(J_{11}-R_{11}\right) Q_{11}-E_{11} K_{11}\right] x_{1}+(\hat{B}-\hat{P}) u, \quad x_{1}\left(t_{0}\right)=x_{1,0} \\
y & =(\hat{B}+\hat{P})^{T} Q_{11} x_{1}+(\hat{S}+\hat{N}) u
\end{aligned}
$$

with Hamiltonian $\hat{\mathcal{H}}\left(x_{1}\right)=\frac{1}{2} x_{1}^{T} Q_{11}^{T} E_{11} x_{1}=\mathcal{H}(x)$, and coefficients

$$
\begin{aligned}
\hat{B} & =B_{1}-\frac{1}{2}\left(J_{21}^{T}-R_{12}\right) L_{22}^{-T}\left(B_{2}+P_{2}\right), \\
\hat{P} & =P_{1}-\frac{1}{2}\left(J_{21}^{T}-R_{12}\right) L_{22}^{-T}\left(B_{2}+P_{2}\right), \\
\hat{S} & =S-\frac{1}{2}\left[\left(B_{2}+P_{2}\right)^{T} L_{22}^{-1}\left(B_{2}-P_{2}\right)+\left(B_{2}-P_{2}\right)^{T} L_{22}^{-T}\left(B_{2}+P_{2}\right)\right] \\
\hat{N} & =N-\frac{1}{2}\left[\left(B_{2}+P_{2}\right)^{T} L_{22}^{-1}\left(B_{2}-P_{2}\right)-\left(B_{2}-P_{2}\right)^{T} L_{22}^{-T}\left(B_{2}+P_{2}\right)\right] .
\end{aligned}
$$

Furthermore, the second part of the state $x_{2}$ is uniquely determined by the algebraic constraint

$$
L_{22} Q_{22} x_{2}=-L_{21} Q_{11} x_{1}-\left(B_{2}-P_{2}\right) u,
$$

and there is a consistency constraint for the initial condition

$$
L_{22}\left(t_{0}\right) Q_{22}\left(t_{0}\right) x_{2}\left(t_{0}\right)=-\left[\left(L_{21}\left(t_{0}\right)\right) Q_{11}\left(t_{0}\right)\right] x_{1}\left(t_{0}\right)-\left(B_{2}\left(t_{0}\right)-P_{2}\left(t_{0}\right)\right) u\left(t_{0}\right) .
$$


Proof. Equation (25) follows directly from the second state equation in (23). Since $\hat{B}-\hat{P}=$ $B_{1}-P_{1}$, we see that $x_{1}$ satisfies the state equation in (24). The output equation is obtained directly by substituting (25) in the output equation of (23).

It remains to prove that (24) is port-Hamiltonian. Since (23) is a pHDAE system, it follows that

$$
Q_{11}^{T} E_{11}=E_{11}^{T} Q_{11}
$$

and

$$
\begin{aligned}
\frac{d}{d t} Q_{11}^{T} E_{11} & =Q_{11}^{T}\left[E_{11} K_{11}-J_{11} Q_{11}\right]+\left[E_{11} K_{11}-J_{11} Q_{11}\right]^{T} Q_{11}, \\
0 & =-Q_{11}^{T}\left(J_{12}+J_{21}^{T}\right) Q_{22}+Q_{11}^{T} E_{11} K_{12}, \\
0 & =Q_{22}^{T} J_{22} Q_{22}+Q_{22}^{T} J_{22}^{T} Q_{22} .
\end{aligned}
$$

Combining (27) with the first equation of (28) gives that the operator $Q_{11}^{T} E_{11} \frac{d}{d t}-Q_{11}^{T}\left(J_{11} Q_{11}-\right.$ $\left.E_{11} K_{11}\right)$ is skew-adjoint.

Furthermore, since $\hat{S}$ is symmetric and $\hat{N}$ is skew-symmetric, system (24) is of the form (5), and thus Theorem 15 gives that (15) is satisfied. So

$$
\frac{d}{d t} \hat{\mathcal{H}}\left(x_{1}\right)=\frac{d}{d t} x_{1}^{T} Q_{11}^{T} E_{11} x_{1}=y^{T} u-\left[\begin{array}{c}
x_{1} \\
u
\end{array}\right]^{T} \hat{W}\left[\begin{array}{c}
x_{1} \\
u
\end{array}\right]
$$

with

$$
\hat{W}=\left[\begin{array}{cc}
Q_{11}^{T} R_{11} Q_{11} & Q_{11}^{T} \hat{P} \\
\hat{P}^{T} Q_{11} & \hat{S}
\end{array}\right] .
$$

On the other hand, since (5) is a pHDAE system, we have that

$$
\frac{d}{d t} \mathcal{H}(x)=y^{T} u-\left[\begin{array}{l}
x \\
u
\end{array}\right]^{T} W\left[\begin{array}{l}
x \\
u
\end{array}\right]
$$

where

$$
W=\left[\begin{array}{ccc}
Q_{11} & 0 & 0 \\
0 & Q_{22} & 0 \\
0 & 0 & I
\end{array}\right]^{T}\left[\begin{array}{ccc}
R_{11} & R_{12} & P_{1} \\
R_{12}^{T} & R_{22} & P_{2} \\
P_{1}^{T} & P_{2}^{T} & S
\end{array}\right]\left[\begin{array}{ccc}
Q_{11} & 0 & 0 \\
0 & Q_{22} & 0 \\
0 & 0 & I
\end{array}\right]
$$

We know that for the same input and initial state with $x_{2}\left(t_{0}\right)$ satisfying $(26)$ the solutions of the two systems are the same, and furthermore we have that

$$
\mathcal{H}(x)=x^{T} Q^{T} E x=x_{1}^{T} Q_{11}^{T} E_{11} x_{1}=\hat{\mathcal{H}}\left(x_{1}\right),
$$

and that (25) holds. Thus, from (29) and (30) we obtain that

$$
\left[\begin{array}{c}
x_{1} \\
u
\end{array}\right]^{T} \hat{W}\left[\begin{array}{c}
x_{1} \\
u
\end{array}\right]=\left[\begin{array}{c}
x_{1} \\
x_{2} \\
u
\end{array}\right]^{T} W\left[\begin{array}{c}
x_{1} \\
x_{2} \\
u
\end{array}\right]=\left[\begin{array}{c}
x_{1} \\
u
\end{array}\right]^{T} W_{X}\left[\begin{array}{c}
x_{1} \\
u
\end{array}\right],
$$

where $W_{X}=X^{T} W X$ with

$$
X=\left[\begin{array}{cc}
I & 0 \\
-Q_{22}^{-1}\left(L_{22}^{-1}\left(J_{21}-R_{12}^{T}\right) Q_{11}\right) & -Q_{22}^{-1} L_{22}^{-1}\left(B_{2}-P_{2}\right) \\
0 & I
\end{array}\right] .
$$


Since (31) has to hold for all $x_{1}$ and $u$, we find that $\hat{W}=W_{X}$, which could also be obtained by straightforward (but tedious) calculation. Since $W$ is symmetric positive semidefinite, so is $\hat{W}$, and hence the reduced system in $x_{1}$ is still port-Hamiltonian with Hamiltonian $\hat{\mathcal{H}}\left(x_{1}\right)$. 口

Note that for the numerical integration or in the control context, as for general DAEs, it is sufficient to carry out the transformation with pointwise orthogonal $\tilde{U}$ from the left and the insertion of $I=\tilde{U} \tilde{U}^{T}$ before $Q$. In this way a differentiation of a computed transformation matrix can be avoided and the pHDAE structure is preserved nonetheless. However, no explicit separation of the parts $x_{1}$ and $x_{2}$ would be obtained in this way and this separation has to be carried out by the numerical solver in the context of the numerical integration method.

Remark 23 For nonlinear pHDAE systems with differentiation-index at most one $(\mu=0)$, the corresponding local result follows directly via the implicit function theorem and application of Theorem 22 to the linearization.

\section{Regularization of higher index pHDAE systems}

In this section we discuss how to modify the regularization procedure discussed for general DAEs in Section 4 to preserve the pHDAE structure. Again, we consider the linear timevarying case (5) and set $L:=J-R$. Suppose that the state equation with $u=0$ already satisfies Hypothesis 21, so that as discussed in Section 4, no reinterpretation of variables or initial feedbacks are necessary. It has been shown in [7] that the extra constraint equations (hidden constraints) that arise from derivatives are uncontrollable, because otherwise the index reduction could have been done via feedback. This means that these extra (uncontrollable) constraint equations are of the form $\hat{A}_{3} x=0$ which corresponds to $\hat{F}_{3}(t, x)=0$ in the nonlinear case, see (20). We add just these constraint equations to our original pHDAE and obtain an overdetermined strangeness-free system, see [30].

Let us make the weak assumption that $E(t)$ has constant rank. This is a restriction that, however, holds in all examples that we have encountered so far, and it can be removed by considering the system in a piecewise fashion, see [30]. Then there exist real orthogonal matrix functions $U_{1}, V_{1} \in C^{1}\left(\mathbb{I}, \mathbb{R}^{n, n}\right)$ such that

$$
U_{1}^{T} E V_{1}=\left[\begin{array}{cc}
\tilde{E}_{11} & 0 \\
0 & 0
\end{array}\right]=: \tilde{E}
$$

with pointwise invertible $\tilde{E}_{11}$.

Perform a transformation of the pHDAE (5) as in Theorem 18 and also form $\hat{A}_{3} V_{1}=$ $\left[\begin{array}{ll}\hat{A}_{31} & \hat{A}_{32}\end{array}\right]$ partitioned accordingly. Since the equations $\hat{A}_{3} x=0$ do not have a control, they cannot contribute to making the system strangeness-free via feedback, so these equations include all the equations that are needed to make the system strangeness-free. Since $\tilde{E}_{11}$ is invertible these extra equations must arise from the full row-rank part of $\hat{A}_{32}$, which we assume to be of constant rank (by considering the problem piecewise if necessary). Then there exist real orthogonal matrix functions $U_{3}$ and $V_{2}$ such that

$$
U_{3}^{T} \hat{A}_{32} V_{2}=\left[\begin{array}{cc}
0 & A_{33} \\
0 & 0
\end{array}\right]
$$


with $A_{33}$ pointwise invertible. The equations in $U_{3}^{T} \hat{A}_{3} x=0$ corresponding to the second row of this factorization cannot contribute to making the system strangeness-free, so they can be just omitted. We may therefore assume that $\hat{A}_{32}$ has full row rank, and that $\hat{A}_{32} V_{2}=\left[\begin{array}{ll}0 & A_{33}\end{array}\right]$ with $A_{33}$ pointwise invertible. Performing a change of variables of the pHDAE with $U_{1}$ and

$$
V:=V_{1}\left[\begin{array}{cc}
I & 0 \\
0 & V_{2}
\end{array}\right]\left[\begin{array}{ccc}
I & 0 & 0 \\
0 & I & 0 \\
-\hat{A}_{31} A_{33}^{-1} & 0 & I
\end{array}\right]
$$

we obtain a pHDAE of the form

$$
\begin{aligned}
\tilde{E}\left[\begin{array}{c}
\dot{x}_{1} \\
\dot{x}_{2} \\
\dot{x}_{3}
\end{array}\right] & =\tilde{L} \tilde{Q}\left[\begin{array}{l}
x_{1} \\
x_{2} \\
x_{3}
\end{array}\right]-\tilde{E} \tilde{K}\left[\begin{array}{l}
x_{1} \\
x_{2} \\
x_{3}
\end{array}\right]+(\tilde{B}-\tilde{P}) u \\
y & =(\tilde{B}+\tilde{P})^{T} \tilde{Q}\left[\begin{array}{l}
x_{1} \\
x_{2} \\
x_{3}
\end{array}\right]+(S+N) u,
\end{aligned}
$$

where $\tilde{K}=V^{-1}(K V+\dot{V}), \tilde{L}=U_{1}^{T} L U_{1}, \tilde{Q}=U_{1}^{T} Q V, \tilde{B}=U_{1}^{T} B$, and $\tilde{P}=U_{1}^{T} P$, together with the constraint $0=A_{33} x_{3}$, i. e. $x_{3}=0$.

Partition

$$
\tilde{Q}=\left[\begin{array}{lll}
\tilde{Q}_{11} & \tilde{Q}_{12} & \tilde{Q}_{13} \\
\tilde{Q}_{21} & \tilde{Q}_{22} & \tilde{Q}_{23} \\
\tilde{Q}_{31} & \tilde{Q}_{32} & \tilde{Q}_{33}
\end{array}\right]
$$

and sssume further that the matrix function

$$
\left[\begin{array}{ll}
\tilde{Q}_{11} & \tilde{Q}_{12} \\
\tilde{Q}_{21} & \tilde{Q}_{22} \\
\tilde{Q}_{31} & \tilde{Q}_{32}
\end{array}\right]
$$

has constant rank. There exists a pointwise real orthogonal matrix function $U_{2}$ such that

$$
U_{2}^{T}\left[\begin{array}{ccc}
\tilde{Q}_{11} & \tilde{Q}_{12} & \tilde{Q}_{13} \\
\tilde{Q}_{21} & \tilde{Q}_{22} & \tilde{Q}_{23} \\
\tilde{Q}_{31} & \tilde{Q}_{32} & \tilde{Q}_{33}
\end{array}\right]=\left[\begin{array}{ccc}
Q_{11} & Q_{12} & Q_{13} \\
Q_{21} & Q_{22} & Q_{23} \\
0 & 0 & Q_{33}
\end{array}\right]
$$

Transforming the pHDAE (32) with $U_{2}$ and $I$ we get a pHDAE of the form

$$
\begin{gathered}
{\left[\begin{array}{lll}
E_{11} & 0 & 0 \\
E_{21} & 0 & 0 \\
E_{31} & 0 & 0
\end{array}\right]\left[\begin{array}{l}
\dot{x}_{1} \\
\dot{x}_{2} \\
\dot{x}_{3}
\end{array}\right]=\left[\begin{array}{lll}
L_{11} & L_{12} & L_{13} \\
L_{21} & L_{22} & L_{23} \\
L_{31} & L_{32} & L_{33}
\end{array}\right]\left[\begin{array}{ccc}
Q_{11} & Q_{12} & Q_{13} \\
Q_{21} & Q_{22} & Q_{23} \\
0 & 0 & Q_{33}
\end{array}\right]\left[\begin{array}{l}
x_{1} \\
x_{2} \\
x_{3}
\end{array}\right]} \\
-\left[\begin{array}{lll}
E_{11} & 0 & 0 \\
E_{21} & 0 & 0 \\
E_{31} & 0 & 0
\end{array}\right]\left[\begin{array}{lll}
K_{11} & K_{12} & K_{13} \\
K_{21} & K_{22} & K_{23} \\
K_{31} & K_{32} & K_{33}
\end{array}\right]\left[\begin{array}{l}
x_{1} \\
x_{2} \\
x_{3}
\end{array}\right]+\left[\begin{array}{c}
B_{1}-P_{1} \\
B_{2}-P_{2} \\
B_{3}-P_{3}
\end{array}\right] u \\
y=\left[\begin{array}{lll}
\left(B_{1}+P_{1}\right)^{T} & \left(B_{2}+P_{2}\right)^{T}\left(B_{3}+P_{3}\right)^{T}
\end{array}\right]\left[\begin{array}{ccc}
Q_{11} & Q_{12} & Q_{13} \\
Q_{21} & Q_{22} & Q_{23} \\
0 & 0 & Q_{33}
\end{array}\right]\left[\begin{array}{l}
x_{1} \\
x_{2} \\
x_{3}
\end{array}\right] \\
+(S+N) u,
\end{gathered}
$$


together with the constraint $0=x_{3}$.

By Theorem 18, system (33) is still a pHDAE system and the Hamiltonian is unchanged. Furthermore, adding the constraint $x_{3}=0$ does not change the solution since it describes all the uncontrollable equations of higher index, and with this constraint system (33) is strangeness-free. Thus we have that the subsystem given by the first two block rows of (33) (which by construction is also port-Hamiltonian) is an index at most one pHDAE which (together with output equation) has the form

$$
\begin{aligned}
{\left[\begin{array}{ll}
E_{11} & 0 \\
E_{21} & 0
\end{array}\right]\left[\begin{array}{l}
\dot{x}_{1} \\
\dot{x}_{2}
\end{array}\right] } & =\left[\begin{array}{ll}
L_{11} & L_{12} \\
L_{21} & L_{22}
\end{array}\right]\left[\begin{array}{ll}
Q_{11} & Q_{12} \\
Q_{21} & Q_{22}
\end{array}\right]\left[\begin{array}{l}
x_{1} \\
x_{2}
\end{array}\right] \\
& -\left[\begin{array}{ll}
E_{11} & 0 \\
E_{21} & 0
\end{array}\right]\left[\begin{array}{ll}
K_{11} & K_{12} \\
K_{21} & K_{22}
\end{array}\right]\left[\begin{array}{l}
x_{1} \\
x_{2}
\end{array}\right]+\left[\begin{array}{l}
B_{1}-P_{1} \\
B_{2}-P_{2}
\end{array}\right] u \\
y & =\left[\begin{array}{ll}
\left(B_{1}+P_{1}\right)^{T} & \left(B_{2}+P_{2}\right)^{T}
\end{array}\right]\left[\begin{array}{ll}
Q_{11} & Q_{12} \\
Q_{21} & Q_{22}
\end{array}\right]\left[\begin{array}{l}
x_{1} \\
x_{2}
\end{array}\right] \\
& +(S+N) u
\end{aligned}
$$

To this system we can apply the results of the previous section and obtain that the system can be further reduced to an implicit standard $\mathrm{pH}$ system.

Example 24 To illustrate the regularization procedure consider again the semidiscretized Example 11. In this example we know directly from the structure what the constraints are and how the procedure can be carried out analytically, since the system is almost in the form that would be obtained from the derivative array. For this reason we present a simplified version of the regularization procedure. It has been shown in [16] that for a (permuted) singular value decomposition (SVD) of $\tilde{N}^{T}$

$$
\tilde{N}^{T}=U_{N}^{T}\left[\begin{array}{c}
0 \\
\Sigma
\end{array}\right] V_{N}
$$

with real orthogonal matrices $U_{N}, V_{N}$ and a nonsingular diagonal matrix $\Sigma \in \mathbb{R}^{n_{3}, n_{3}}$. Transforming (10) with $U=V=\operatorname{diag}\left(I, U_{N}^{T}, V_{N}^{T}\right)$, and setting $U_{N} x_{2}=\left[\begin{array}{ll}x_{2,2}^{T} & x_{2,3}^{T}\end{array}\right]^{T}, V_{N} x_{3}=\tilde{x}_{3}$, as well as $x_{2}^{0}=V_{N}\left[\begin{array}{lll}x_{2,2}^{0}{ }^{T} & x_{2,3}^{0}{ }^{T}\end{array}\right]^{T}$ we obtain a transformed system

$$
\left[\begin{array}{cccc}
M_{1} & 0 & 0 & 0 \\
0 & M_{2,2} & M_{2,3} & 0 \\
0 & M_{2,3}^{T} & M_{3,3} & 0 \\
0 & 0 & 0 & 0
\end{array}\right] \frac{d}{d t}\left[\begin{array}{c}
x_{1} \\
x_{2,2} \\
x_{2,3} \\
\tilde{x}_{3}
\end{array}\right]+\left[\begin{array}{cccc}
0 & G_{1,2} & G_{1,3} & 0 \\
-G_{1,2}^{T} & D_{2,2} & D_{2,3} & 0 \\
-G_{1,3}^{T} & D_{2,3}^{T} & D_{3,3} & -\Sigma \\
0 & 0 & \Sigma & 0
\end{array}\right]\left[\begin{array}{c}
x_{1} \\
x_{2,2} \\
x_{2,3} \\
\tilde{x}_{3}
\end{array}\right]=\left[\begin{array}{c}
0 \\
B_{2,2} \\
B_{3,2} \\
0
\end{array}\right] u
$$

In this form the noncontrollable index two constraints are given by the fourth block row and it follows immediately that $x_{2,3}=0$. which in particular the initial condition $x_{2,3}^{0}$ has to satisfy. The vectors $x_{1}, x_{2,2}$ are solutions of the implicit ordinary $\mathrm{pH}$ system

$$
\left[\begin{array}{cc}
M_{1} & 0 \\
0 & M_{2,2}
\end{array}\right] \frac{d}{d t}\left[\begin{array}{c}
x_{1} \\
x_{2,2}
\end{array}\right]+\left[\begin{array}{cc}
0 & G_{1,2} \\
-G_{1,2}^{T} & D_{2,2}
\end{array}\right]\left[\begin{array}{c}
x_{1} \\
x_{2,2}
\end{array}\right]=\left[\begin{array}{c}
0 \\
B_{2,2}
\end{array}\right] u
$$

with initial conditions $x_{1}(0)=x_{1}^{0}, x_{2,2}(0)=x_{2,2}^{0}$, so they are well-defined continuously differentiable functions for any piecewise continuous $u$ and any choice of the initial conditions. 
Finally we get the component $x_{3}$ (the Lagrange multiplier) via

$$
x_{3}=V_{N}^{T} \Sigma^{-1}\left(M_{2,3}^{T} \frac{d}{d t} x_{2,2}-G_{1,3}^{T} x_{1}+D_{2,3}^{T} x_{2,2}-B_{3,2} u\right),
$$

and this is the implicit index one constraint in the DAE. Since both type of (the explicit and the hidden) constraints have to be satisfied for the initial condition, it means that the transformed initial condition also has to satisfy the consistency condition

$$
x_{3}(0)=V_{N}^{T} \Sigma^{-1}\left(M_{2,3}^{T} \frac{d}{d t} x_{2,2}(0)-G_{1,3}^{T} x_{1}(0)+D_{2,3}^{T} x_{2,2}(0)-B_{3,2} u(0)\right)
$$

Condition (38) leads to a relationship between the input $u$ and the state at $t=0$, which is a constraint that has to be satisfied to have a classical solution. Furthermore, we see immediately that to obtain a continuous $x_{3}$ the function $B_{3,2} u$ has to be continuous and $u$ has to be such that $B_{2,2} u$ leads to a continuous $M_{2,3}^{T} \frac{d}{d t} x_{2,2}$. The implicit ordinary $\mathrm{pH}$ system (36) describes the dynamics of the system, while the other two equations describe the constraints.

Remark 25 For nonlinear pHDAE systems satisfying Hypothesis 21 with $\mu>0$, the corresponding local result follows directly via linearization and the implicit function theorem.

\section{Conclusion}

A new definition of port-Hamiltonian descriptor systems has been introduced. It has been shown that this formulation retains the classical properties of port-Hamiltonian systems, such as the dissipation inequality and invariance under Galerkin projection, that it is invariant under time-varying changes of basis and that it is valid also for DAEs of differentiationindex larger than one. It has been demonstrated that under some additional weak constantrank assumptions, any such pHDAE can be reformulated as an implicitly defined standard $\mathrm{PH}$ system plus an algebraic constraint that describes the manifold where the dynamics of the system takes place and that also describes the consistent initial conditions. Just as for standard DAEs, the reformulated system is well suited for numerical integration and control, since all constraints are available.

\section{Acknowledgments}

We acknowledge many interesting discussions with Robert Altmann and Philipp Schulze from TU Berlin and Arjan Van der Schaft from RU Groningen. We also thank two anonymous reviewers for helpful comments to improve the presentation. The first author has been supported by Einstein Foundation Berlin, through an Einstein Visiting Fellowship. The second author has been supported by Deutsche Forschungsgemeinschaft for Research support via Project A02 in CRC 1029 TurbIn, and project B03 in CRC-TR154 as well as by Einstein Foundation Berlin within the Einstein Center ECMath.

\section{References}

[1] J. Bals, G. Hofer, A. Pfeiffer, and C. Schallert. Virtual iron bird - A multidisciplinary modelling and simulation platform for new aircraft system architectures. In Deutscher Luft- und Raumfahrtkongress, Friedrichshafen, Germany, 2005. 
[2] C. Beattie and S. Gugercin. Structure-preserving model reduction for nonlinear portHamiltonian systems. In 50th IEEE Conference on Decision and Control and European Control Conference (CDC-ECC), 2011, pages 6564-6569. IEEE, 2011.

[3] A. Binder, V. Mehrmann, A. Miedlar, and P. Schulze. A Matlab toolbox for the regularization of descriptor systems arising from generalized realization procedures. Preprint 24-2015, Institut für Mathematik, TU Berlin, 2015.

[4] P. C. Breedveld. Modeling and Simulation of Dynamic Systems using Bond Graphs, pages 128-173. EOLSS Publishers Co. Ltd./UNESCO, Oxford, UK, 2008.

[5] K. E. Brenan, S. L. Campbell, and L. R. Petzold. Numerical Solution of Initial-Value Problems in Differential Algebraic Equations. SIAM Publications, Philadelphia, PA, 2nd edition, 1996.

[6] A. Bunse-Gerstner, R. Byers, V. Mehrmann, and N. K. Nichols. Feedback design for regularizing descriptor systems. Linear Algebra Appl., 299:119-151, 1999.

[7] R. Byers, P. Kunkel, and V. Mehrmann. Regularization of linear descriptor systems with variable coefficients. SIAM J. Control Optim., 35(1):117-133, 1997.

[8] C. I. Byrnes, A. Isidori, and J. C. Willems. Passivity, feedback equivalence, and the global stabilization of minimum phase nonlinear systems. IEEE Trans. Autom. Control, 36:1228-1240, 1991.

[9] S. L. Campbell. A general form for solvable linear time varying singular systems of differential equations. SIAM J. Math. Anal., 18:1101-1115, 1987.

[10] S. L. Campbell. Linearization of DAEs along trajectories. Z. Angew. Math. Phys., 46:70-84, 1995.

[11] S. L. Campbell, P. Kunkel, and V. Mehrmann. Regularization of linear and nonlinear descriptor systems. In L. T. Biegler, S. L. Campbell, and V. Mehrmann, editors, Control and Optimization with Differential-Algebraic Constraints, Advances in Control and Design, chapter 2, pages 17-36. SIAM Publications, 2012.

[12] F. Couenne, C. Jallut, B. M. Maschke, M. Tayakout, and P. C. Breedveld. Bond graph for dynamic modelling in chemical engineering. Chemical engineering and processing, Elsevier, Amsterdam, 47:1994-2003, 2008.

[13] L. Dai. Singular Control Systems, volume 118 of Lecture Notes in Control and Inform. Sci. Springer-Verlag, Berlin/Heidelberg, 1989.

[14] P.A.M. Dirac. Generalized Hamiltonian dynamics. Canadian J. Math, 2:129-148, 1950.

[15] H. Egger and T. Kugler. Damped wave systems on networks: Exponential stability and uniform approximations. Numerische Mathematik, 138(4):839-867, 2018.

[16] H. Egger, T. Kugler, B. Liljegren-Sailer, N. Marheineke, and V. Mehrmann. On structure preserving model reduction for damped wave propagation in transport networks. SIAM J. Sci. Comput., 40:A331-A365, 2018. 
[17] E. Eich-Soellner and C. Führer. Numerical Methods in Multibody Dynamics. Teubner, Stuttgart, 1998.

[18] R. W Freund. The sprim algorithm for structure-preserving order reduction of general RLC circuits. In Model reduction for circuit simulation, pages 25-52. Springer, 2011.

[19] K. Fujimoto and T. Sugie. Canonical transformation and stabilization of generalized Hamiltonian systems. Systems \& Control Letters, 42(3):217-227, 2001.

[20] N. Gillis, V. Mehrmann, and P. Sharma. Computing the nearest stable matrix pairs. Numer. Lin. Alg. Appl., To appear, 2018.

[21] G. Golo, A. J. van der Schaft, P. C. Breedveld, and B. M. Maschke. Hamiltonian formulation of bond graphs. In A. Rantzer R. Johansson, editor, Nonlinear and Hybrid Systems in Automotive Control, pages 351-372. Springer, Heidelberg, 2003.

[22] S. Gugercin, R. V. Polyuga, C. Beattie, and A. J. van der Schaft. Structure-preserving tangential interpolation for model reduction of port-Hamiltonian systems. Automatica, 48:1963-1974, 2012.

[23] M. Hiller and K. Hirsch. Multibody system dynamics and mechatronics. Z. Angew. Math. Mech., 86(2):87-109, 2006.

[24] D. Hinrichsen and A. J. Pritchard. Mathematical Systems Theory I. Modelling, State Space Analysis, Stability and Robustness. Springer-Verlag, New York, NY, 2005.

[25] M. Hou. A three-link planar manipulator model. Sicherheitstechnische Regelungs- und Meßtechnik, Bergische Universität-GH Wuppertal, Germany, May 1994.

[26] M. Hou and P. C. Müller. $L Q$ and tracking control of descriptor systems with application to constrained manipulator. Technical report, Sicherheitstechnische Regelungs- und Meßtechnik, Universität Wuppertal, Gauß-Straße 20, D-5600 Wuppertal 1, Germany, 1994.

[27] B. Jacob and H. Zwart. Linear port-Hamiltonian systems on infinite-dimensional spaces. Operator Theory: Advances and Applications, 223. Birkhäuser/Springer Basel AG, Basel CH, 2012.

[28] C. Kleijn. 20-sim 4C 2.1 Reference manual. Controlab Products B.V., 2013.

[29] P. Kunkel and V. Mehrmann. Analysis of over- and underdetermined nonlinear differential-algebraic systems with application to nonlinear control problems. Math. Control Signals Systems, 14:233-256, 2001.

[30] P. Kunkel and V. Mehrmann. Differential-Algebraic Equations. Analysis and Numerical Solution. EMS Publishing House, Zürich, Switzerland, 2006.

[31] P. Kunkel, V. Mehrmann, and W. Rath. Analysis and numerical solution of control problems in descriptor form. Math. Control Signals Systems, 14:29-61, 2001.

[32] P. Kunkel, V. Mehrmann, and L. Scholz. Self-adjoint differential-algebraic equations. Math. Control Signals Syst., 26:47-76, 2014. 
[33] R. Lamour, R. März, and C. Tischendorf. Differential-algebraic equations: a projector based analysis. Springer Science \& Business Media, 2013.

[34] B. M. Maschke, A. J. van der Schaft, and P. C. Breedveld. An intrinsic Hamiltonian formulation of network dynamics: non-standard Poisson structures and gyrators. $J$. Franklin Inst., 329:923-966, 1992.

[35] C. Mehl, V. Mehrmann, and M. Wojtylak. Linear algebra properties of dissipative Hamiltonian descriptor systems. Preprint 01-2018, MATHEON, DFG Research Center Mathematics for Key Technologies in Berlin, 2018.

[36] V. Mehrmann and C. Schröder. Nonlinear eigenvalue and frequency response problems in industrial practice. J. Math. Ind., 1:18, 2011.

[37] R. Ortega, A. J. van der Schaft, Y. Mareels, and B. M. Maschke. Putting energy back in control. Control Syst. Mag., 21:18-33, 2001.

[38] J. W. Polderman and J. C. Willems. Introduction to Mathematical Systems Theory: A Behavioural Approach. Springer-Verlag, New York, NY, 1998.

[39] R. V. Polyuga and A. J. van der Schaft. Structure preserving model reduction of portHamiltonian systems by moment matching at infinity. Automatica, 46:665-672, 2010.

[40] A. J. van der Schaft. Port-Hamiltonian systems: network modeling and control of nonlinear physical systems. In Advanced Dynamics and Control of Structures and Machines, CISM Courses and Lectures, Vol. 444. Springer Verlag, New York, N.Y., 2004.

[41] A. J. van der Schaft. Port-Hamiltonian systems: an introductory survey. In J. L. Verona M. Sanz-Sole and J. Verdura, editors, Proc. of the International Congress of Mathematicians, vol. III, Invited Lectures, pages 1339-1365, Madrid, Spain, 2006.

[42] A. J. van der Schaft. Port-Hamiltonian differential-algebraic systems. In Surveys in Differential-Algebraic Equations I, pages 173-226. Springer-Verlag, 2013.

[43] A. J. van der Schaft and B. M. Maschke. Hamiltonian formulation of distributedparameter systems with boundary energy flow. J. Geom. Phys., 42:166-194, 2002.

[44] W. Schiehlen. Advanced multibody system dynamics. Kluwer Academic Publishers, Stuttgart, Germany, 1993.

[45] K. Schlacher and A. Kugi. Automatic control of mechatronic systems. Int. J. Appl. Math. Comput. Sci., 11(1):131-164, 2001.

[46] L. Scholz. Condensed forms for linear port-Hamiltonian descriptor systems. Preprint 09-2017, Institut für Mathematik, TU Berlin, 2017.

[47] W. Trautenberg. Simpack 8.9. Manual, INTEC GmbH, Angelrieder Feld 13, 82234 Wessling. 